\title{
Estruturação da área de informação em saúde a partir da gerência de recursos informacionais: análise de experiência ${ }^{1}$
}

\author{
Alaneir de Fatima dos Santos, ${ }^{2}$ Janete Maria Ferreira, ${ }^{3}$ \\ Neuslene Rivers Queiroz ${ }^{2}$ e Helvécio Miranda Magalhães Júnior ${ }^{4}$
}

Como citar Santos AF, Ferreira JM, Queiroz NR, Magalhães Júnior HM. Estruturação da área de informação em saúde a partir da gerência de recursos informacionais: análise de experiência. Rev Panam Salud Publica. 2011;29(6):409-15.

RESUMO

Objetivo. Relatar a experiência da Secretaria Municipal de Saúde de Belo Horizonte (SMSA/BH), no Estado de Minas Gerais, Brasil, na aplicação dos conceitos da gerência de recursos informacionais (GRI) para o desenvolvimento de um sistema de informação em saúde. Métodos. Neste estudo de caso foram analisados documentos que descrevem as iniciativas na área de informação, seus mecanismos de estruturação e resultados alcançados com um sistema de gestão focado no prontuário eletrônico, no período de 2003 a 2008. Esse processo é descrito e analisado a partir do conceito de GRI.

Resultados. O sistema contribuiu para a mudança do modelo assistencial a partir da informatização integrada de 103 unidades básicas de saúde, nove unidades especializadas e 503 equipes de saúde da família, com ênfase nas informações relevantes para o programa saúde da família.

Conclusões. Os seguintes componentes da GRI foram aplicados: estruturação de um locus único para as tecnologias de informação e a área de informação, potencial de alavancagem das tecnologias de informação e fórum estratégico de decisão quanto à informação. A limitação encontrada refere-se à utilização de informação estratégica para a tomada de decisão de longo prazo.

Palavras-chave Sistemas de informação; sistemas de registros médicos computarizados; atenção primária à saude; Brasil.

Dentro do leque de instrumentos necessários à gerência eficaz das organizações, a informação pode ser vista como

1 Conflito de interesses: o grupo de autores deste artigo participou enquanto coordenação/assessorias da área de informação da Secretaria Municipal de Saúde de Belo Horizonte (SMSA/BH) no período analisado.

2 Universidade Federal de Minas Gerais (UFMG), Departamento de Medicina Preventiva e Social, Belo Horizonte (MG), Brasil. Correspondência: Alaneir de Fatima dos Santos, laines@uol.com.br

3 SMSA/BH, Gerência de Tecnologia e Informação em Saúde, Belo Horizonte (MG), Brasil.

4 Ministério da Saúde, Secretaria de Assistência à Saúde, Gabinete do Secretário, Brasília (DF), Brasil. um insumo de importância capital. Alguns autores $(1,2)$ apontam a informação como insumo que pode, gradativamente, substituir o capital como recurso estratégico das empresas e contribuir para a criação da nova sociedade da informação.

Da convergência dos termos informação e tecnologias de informação origina-se o termo gerência de recursos informacionais (GRI), que associa a institucionalização das tecnologias de computador, como recursos organizacionais, aos efeitos das tecnologias da comuni- cação e informação na sociedade, reconhecendo-os como críticos para a sociedade pós-industrial (3). A GRI surgiu nos anos 1970 como conceito (4) que pode ser considerado multidisciplinar, advindo da experiência de gerenciar registros (cujo principal objetivo é facilitar o acesso aos documentos), dados (muitas vezes considerados informação, mas que são fatos ou eventos processados por máquinas e pessoas) e informação propriamente dita.

A GRI tem sido definida como: planejamento, gerenciamento, previsão orçamentária, organização, direcionamento, 
treinamento e controle associados com informação governamental, incluindo tanto a informação per se quanto os recursos relacionados, tais como pessoal, recursos financeiros e tecnologia (5); o processo de identificar, adquirir, integrar e aplicar de modo eficiente e efetivo os recursos de informação para localizar necessidades de informação atuais e futuras (6); uma função gerencial que serve para desenvolver políticas, programas e procedimentos para planejar, administrar e controlar pedidos de informação e apoiar recursos manipulados de informação (7). Stewart (8) sintetiza o conceito: "GRI é a maneira eficaz de tratar integrativamente a informação externa e interna para uso estratégico pelos tomadores de decisão nas organizações, visando a otimizar a performance dessas instituições e sintonizá-las com o ambiente externo" (p. 01).

A partir de 1998, a discussão de GRI passou a incorporar formulações advindas da gestão do conhecimento, que destaca a necessidade de estruturar e gerenciar o conhecimento intangível de uma organização (9). A valorização dessa dimensão se deu após a ascensão da reengenharia de processos, que possuía ênfase exacerbada em incorporação de tecnologias da informação (10), desconhecendo as especificidades da organização dos processos de trabalho de uma determinada instituição e as potencialidades do uso contínuo de informações (11). Atualmente, a ênfase deslocou-se para a questão da competência informacional em suas distintas dimensões, assim como para a estruturação de metodologias que possam contribuir para um processo de tomada de decisões informado por parte de níveis diretivos (12).

O uso prático do conceito GRI tem sido verificado nos setores governamental, militar e na iniciativa privada. A partir de 2002, a área de informação da Secretaria Municipal de Saúde de Belo Horizonte (SMSA/BH), no Estado de Minas Gerais, Brasil, passou a estruturar-se tendo como diretriz fundamental o conceito de GRI.

Belo Horizonte tem um longo caminho percorrido na estruturação de sistemas de informação em saúde. Desde 1993, houve um esforço de construção de um único instrumento de coleta de dados para os profissionais da rede pública, superando as iniciativas fragmentadoras advindas de demandas do Ministério da
Saúde e dos próprios setores da SMSA/ $\mathrm{BH}$. Essas iniciativas fragmentadoras, presentes em muitos municípios brasileiros, geram um contexto no qual, para um mesmo atendimento realizado pelo profissional, é necessário o preenchimento de diversos instrumentos de coleta de informação, ampliando o tempo gasto para o registro, gerando duplicidades e comprometendo a qualidade da informação gerada. Desenvolveram-se também, especialmente, sistemas para as atividades de controle e avaliação dos setores contratados/conveniados que prestam serviços de alto custo e hospitalares, buscando aperfeiçoar os mecanismos de avaliação de qualidade e controle dos serviços ofertados.

A partir de 1998, foram alocados aos distritos sanitários profissionais diretamente vinculados à área de informação, envolvendo digitadores, pessoal administrativo e profissionais da área de informática e de saúde, que se responsabilizaram pela estruturação dos bancos de dados e pela produção, análise e uso de informações. O processo de estruturação dos bancos de dados abrange ações de configuração de sistemas, controle de senhas de acesso a informações e entrada de dados. Esses profissionais têm se reunido de forma periódica para planejar, implantar projetos e monitorar as atividades da área.

Outra característica do modelo assistencial de Belo Horizonte é a utilização efetiva de georreferenciamento (Sistema de Informações sobre Nascidos Vivos, Sistema de Informações sobre Mortalidade, Sistema de Informação Hospitalar, Cadastro do Cartão SUS, Sistema de Alto Custo). Os sistemas de georreferenciamento utilizam a base cadastral de ruas do Município, estruturada por setor censitário e pela agregação desses setores de acordo com a área de abrangência dos centros de saúde. Essa base cadastral é utilizada para a entrada de dados de endereço dos pacientes em todos esses sistemas. Por exemplo, quando um paciente é internado, é possível informar ao centro de saúde, de forma automática, os dados relativos à internação daquele paciente. Isso permitiu que esses sistemas tivessem capacidade de desagregação de suas variáveis, possibilitando análises por município, distrito sanitário e centro de saúde. Essa característica foi sendo reforçada ao longo do tempo, e, a partir de 2000, todos os sistemas da SMSA/BH já se estruturavam dessa forma.
Como resultado dessas iniciativas, houve uma aproximação entre as áreas de informação e de informática, redundando em atuação integrada ao longo dos anos. Foi nesse contexto que ocorreu no Município, em 2000, a experiência de implantação do programa saúde da família (PSF), em larga escala, com 504 equipes. Por outro lado, também foi significativo o esforço da área de informação para contribuir para a gestão anual do orçamento da SMSA/BH, que, em 2008, ficou próximo de R\$ 1,5 bilhão, sendo que uma grande parte desses recursos é transferida para o setor contratado/conveniado, de forma periódica, como pagamento por atividades realizadas. Até esse momento, Belo Horizonte não tinha tido experiência com o uso de tecnologias de informação na área assistencial - o foco havia sempre sido a informatização de sistemas administrativos e de sistemas de controle do setor contratado/conveniado. Percorria, assim, o caminho clássico de incorporação de recursos de informação na área de saúde — primeiro a área administrativa, depois a assistência (13).

Em 2007, a SMSA/BH adotou uma definição de GRI, expressa no documento de criação da gerência de recursos informacionais (14), que se aproxima de um conceito multidisciplinar, envolvendo quatro elementos básicos: gerência, tecnologia, informação e ambiente. Enquanto "gerência", a GRI pode oferecer "suporte informacional" aos vários níveis decisórios da organização, destacando-se aqueles relacionados ao custo, à qualidade e ao uso eficaz da informação. No que se refere ao elemento "tecnologia", consideram-se as tecnologias da informação disponíveis e que possam apoiar o processo decisório da organização, tais como gestão de hardware e software, comunicação de dados e processos de comunicação eletrônica. Enquanto recurso da organização, o elemento "informação" tem custo e valor, e sua gerência pode aumentar a capacidade de criar e reter o conhecimento organizacional, além de contribuir para a melhoria do processo decisório. No que concerne ao elemento "ambiente", o trabalho da GRI é integrar criticamente os diversos meios internos e externos (pessoas, fontes de informação e tecnologias) para apoiar a gestão organizacional. É a partir desse escopo, advindo do desenvolvimento da sociedade da informação, no qual as ações vinculadas à informação e às tecnologias de informação se 
imbricam de forma cada vez mais orgânica, que a discussão sobre a área de informação na SMSA/BH se processou.

A centralidade do conceito de GRI para essa área conduziu à decisão de incorporar, de forma significativa, tecnologias de informação na rede assistencial em Belo Horizonte - centradas na estruturação de um sistema de gestão em saúde que se baseia no prontuário eletrônico do paciente (PEP). O objetivo deste estudo foi relatar a experiência de implantação do sistema de gestão em saúde baseado no PEP e evidenciar o acerto e a pertinência da posição central da GRI na política de informações da SMSA/BH.

\section{MÉTODOS}

Utilizou-se a metodologia de estudo de caso (15), que possibilita enfatizar a multiplicidade de dimensões de um problema, abordando-o como um todo, e realizar uma análise em profundidade dos processos e das relações entre eles, além de estimular novas descobertas, em função da flexibilidade de planejamento (16). Isso permitiu a identificação dos múltiplos aspectos envolvidos na aplicação concreta do conceito de GRI na estruturação da área de informação da SMSA/BH, bem como dos resultados obtidos.

Foi analisada a estruturação da política de informação da SMSA no período de implantação do sistema informatizado de gestão da área de saúde baseado no PEP, ocorrida nos anos de 2003 a 2008. Para a coleta de dados, foram consultados: atas de reuniões dos comitês de informação da SMSA/BH, do projeto saúde em rede (que conduziu a informatização da secretaria municipal) e da área de epidemiologia e informação; documentos publicados no diário oficial do município, relativos à área de informação; relatórios anuais de gestão; publicações e trabalhos apresentados em congressos da área de epidemiologia e informação/informática. Quando houve necessidade de complementar alguma informação, os grupos técnicos diretivos foram consultados por meio de entrevistas.

Inicialmente, procuramos identificar nos documentos as iniciativas desenvolvidas na área de informação, seus mecanismos de estruturação e o detalhamento de sua implantação. A seguir, foi identificada a principal experiência implantada no período analisado, um sistema informatizado de gestão da área de saúde, focado no PEP. As principais características desse sistema foram sistematizadas, assim como os resultados alcançados com sua implantação, correlacionando-os com a estruturação da área de informação. Esse processo foi descrito e analisado tendo como referência o conceito de GRI.

\section{RESULTADOS}

\section{Estruturação da política de informação da SMSA}

O conceito de GRI para a estruturação da área de informação consolidou-se processualmente, ou seja: a GRI não foi instituída em um primeiro momento; inicialmente, foram estruturados grupos de trabalho envolvendo informática, epidemiologia e informação. Só a partir desses grupos foi estruturada a GRI (figura 1). Como transição para a posterior convergência das iniciativas institucionais na área de informação, criou-se, em 2003, o comitê gestor da informação (17), composto pelas áreas que lidam com informação, informática e níveis diretivos, o que permitiu unidade da ação, com ampla estruturação dos diversos componentes da GRI. Percebe-se no documento que orientou a implantação do comitê gestor a intenção de constituir um locus único, estratégico, de decisões relativas à informação - uma característica fundamental da GRI.
Na perspectiva de integração entre tecnologias, recursos e informação, foram estruturados dois grupos. O primeiro deles, constituído em 2003, teve como objetivo principal acelerar a incorporação de recursos informacionais na rede pública, com foco na área assistencial, particularmente o sistema de gestão de saúde (18), estruturado a partir do PEP. Esse grupo também se responsabilizou pela prospecção tecnológica de inovações na área e pela captação de recursos. Foram estabelecidos contatos contínuos com a Universidade Federal de Minas Gerais (UFMG), através das faculdades de medicina, enfermagem e odontologia e do Laboratório de Computação Científica.

Em 2005, o segundo grupo foi constituído, tendo como foco a gestão de hardware e softwares existentes (19) em uma estrutura composta por $5000 \mathrm{mi}-$ crocomputadores e 3000 impressoras, com redes que interligam mais de 200 unidades de saúde, proporcionando acesso on-line e em tempo real a aproximadamente 80 sistemas, acessados pelos 15000 trabalhadores do Sistema Único de Saúde de Belo Horizonte (SUS-BH). Em 2006, para potencializar a dimensão estratégica da informação, foram levadas a cabo as primeiras iniciativas vinculando a informação ao planejamento da SMSA/BH, com a estruturação de um sistema voltado especificamente para esse fim (20). As áreas de epidemiologia

FIGURA 1. Fases de implantação da gerência de recursos informacionais (GRI) na Secretaria Municipal de Saúde de Belo Horizonte, Minas Gerais, Brasil, 2003 a 2008

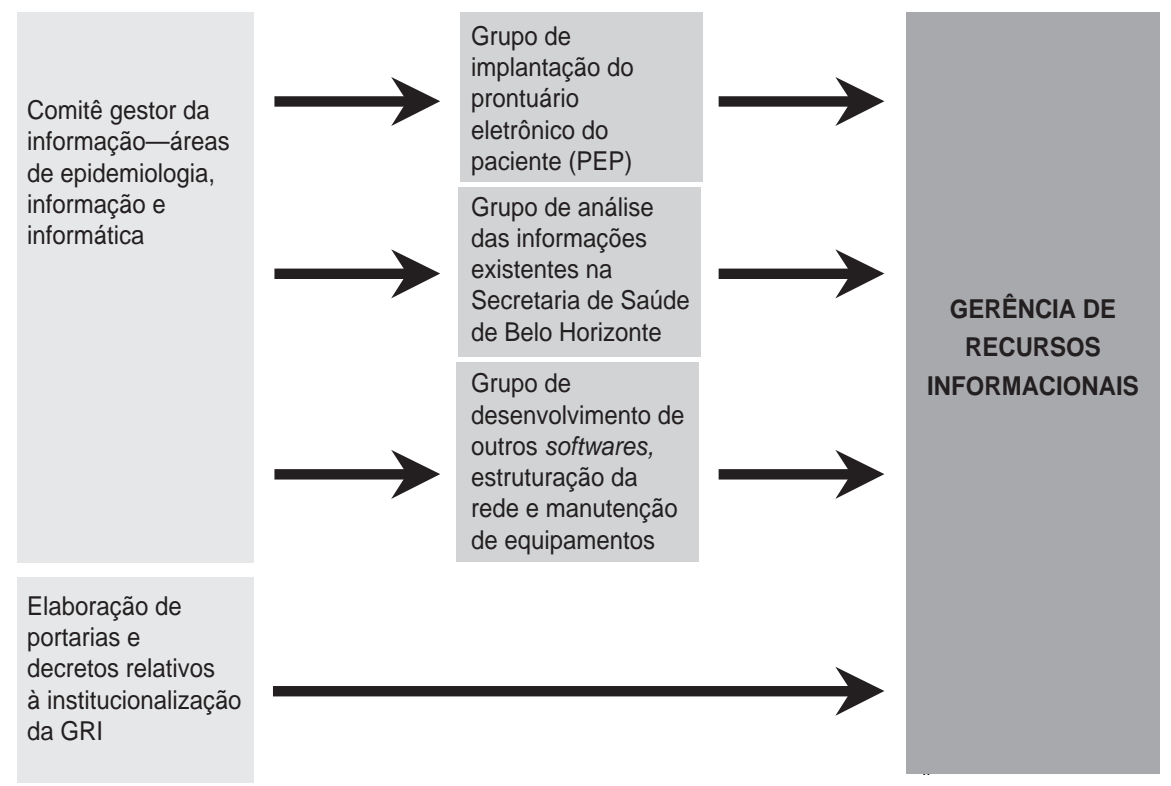


e informação continuaram a analisar os dados disponíveis, gerados pelos sistemas existentes, administrando a transição entre o sistema manual e o sistema assistencial informatizado, o que permitiu a continuidade do acesso à informação pelas unidades de saúde.

Paralelamente a esse processo, foram elaborados marcos institucionais para a futura área de recursos informacionais da SMSA/BH, que se oficializou em 2007, mediante criação da GRI, com os seguintes objetivos: formular, em conjunto com o nível diretivo da instituição, as estratégias de absorção de tecnologia de informação (TI) pela SMSA/BH; implementar as estratégias de absorção de TI, otimizando e direcionando as relações entre os profissionais de saúde dos diversas setores da SMSA/BH e da Empresa de Processamento de Dados de Belo Horizonte (PRODABEL) e estimulando a gestão do conhecimento institucional; e estruturar os distintos processos de uso da informação, de forma a contribuir para a construção de um processo de tomada de decisões que tenha a informação como referência central. Esse documento destacava a intenção de fazer convergirem para esse setor denominado GRI as iniciativas relativas à informação.

\section{Implantação do sistema de gestão em saúde baseado no PEP}

Em Belo Horizonte, a implantação do sistema informatizado de gestão da área de saúde baseado no PEP centrou-se na busca pela qualificação das atividades prestadas aos usuários do serviço de saúde, no sentido de melhorar a assistência, aperfeiçoar os processos gerenciais e monitorar e avaliar os serviços. Com foco no aperfeiçoamento de processos assistenciais executados, foi adquirido no mercado pela SMSA/BH um software comercial de prontuário eletrônico. O levantamento dos requisitos se deu em processo coparticipativo, que envolveu trabalhadores e níveis gerenciais diretamente responsáveis pelas tarefas, em relação estreita e dialógica.

A incorporação desse recurso nos processos de trabalho já existentes requereu estratégias de sensibilização, reflexão sobre a organização do trabalho, parâmetros de qualidade e mudanças no modelo assistencial. A organização do processo de trabalho em cada unidade foi debatida para que os potenciais do sis- tema fossem percebidos; a seguir, o processo de trabalho foi reformulado, utilizando-se o potencial de alavancagem que o recurso informação/tecnologias possui.

Também a partir dessa concepção, buscou-se um processo integrado de gerenciamento com a informática. Foram estruturados grupos técnicos das áreas de saúde e de informática, que tinham autonomia para gerenciar, de forma integrada, todos os recursos que envolviam a área de tecnologia e informação da SMSA/BH e PRODABEL/Saúde. A equipe da PRODABEL - analistas de rede e de sistemas, programadores e técnicos - estruturava-se com coordenação específica, mas subordinada à área de informação da SMSA/BH, tendo sido estabelecidas competências para cada equipe.

O comitê gestor da informação, envolvendo níveis estratégicos da SMSA, reuniu-se de forma periódica durante o projeto de implantação do sistema, tomando decisões relativas às prioridades, articulações necessárias e definindo recursos a serem alocados ao projeto.

\section{Características e componentes do modelo implantado}

O sistema, no final de 2008, encontrava-se implantado em 103 unidades básicas de saúde da rede e em nove unidades especializadas. Haviam sido alocados 2450 computadores para o uso desse sistema, com uma média de 15 computadores por unidade básica de saúde e a realização de 890 acessos simultâneos diariamente (21).

O sistema de gestão de saúde em rede, que continua sendo utilizado pelos profissionais, é uma aplicação desenvolvida em Visual Basic 6.0, adquirida no mercado mas reformulada pela PRODABEL para adaptar-se à realidade da SMSA/ BH. Destina-se às unidades de saúde básicas, especializadas e de urgência, com os seguintes módulos:

- Atualização de cadastro: realiza e atualiza o cadastro dos indivíduos e famílias.

- Acolhimento: registra o atendimento e o encaminhamento executados.

- Agendamento: propicia o agendamento de atividades individuais e coletivas, assegurando o limite de atendimento diário por profissional.
- Recepção: registra pacientes agendados e recepciona os demais, permitindo o cadastramento provisório.

- Atendimento médico: permite ao médico acesso aos diagnósticos e condutas. Engloba história clínica do paciente, história pregressa, solicitação e resultados de exames, prescrição de medicamentos e encaminhamentos. Rastreia os exames solicitados, identificando duplicidades. Possui protocolos: mulher, criança, adulto, hipertensão, diabetes, pré-natal, asma, idoso, saúde mental e acolhimento.

- Registro de atividades coletivas (grupos): permite o agendamento e o registro de atividades coletivas, como por exemplo atividades de grupos operativos (grupos de hipertensos, diabéticos, pré-natal, reuniões de equipe), permitindo a entrada de dados individuais dos pacientes do grupo - níveis pressóricos ou glicêmicos - ou registros gerais.

- Coleta de material biológico: faz o gerenciamento do processo de coleta, com emissão de etiquetas para os tubos, a partir da solicitação de exames informatizados, o que agiliza o atendimento e diminui o tempo de espera do paciente. O módulo possibilita que a unidade tenha acesso ao resultado do exame tão logo seja laudado no laboratório.

- Farmácia: módulo articulado com a prescrição do profissional que permite controle de dispensação de medicamentos por paciente (inclusive com prazo de validade). É integrado ao sistema de materiais e almoxarifado da secretaria e das farmácias distritais, fazendo controle do estoque de medicamentos no centro de saúde e fornecendo ao profissional essas informações.

- Módulo de controle de almoxarifado: integrado ao sistema de materiais geral, permite que a unidade controle o estoque e faça requisições por meio do sistema.

- Módulo de recursos humanos (RH): integrado com o sistema de $\mathrm{RH}$ da SMSA, permite a atualização dos dados funcionais (lotação, cargo, categoria, função, etc.) e a realização de transferência entre unidades, tanto dos trabalhadores da rede própria quanto dos terceirizados.

- Módulo de urgência: é composto por submódulos (recepção, triagem classi- 
ficatória e registro do atendimento), respeitando as especificidades da urgência.

- Módulo extrator de relatórios: relatórios analíticos e sintéticos por microárea, equipe de PSF, unidade de saúde, distrito e município.

- Módulo de indicadores: gera indicadores por equipe de PSF, unidade de saúde e distrito, com dados quantitativos e emissão de listagens. Permite que a equipe do PSF tenha uma visão completa do seu desempenho; centrase em ações de prevenção, com capacidade de listar os pacientes faltosos e indicar o endereço dos mesmos por equipe do PSF. Direciona a atividade dos agentes comunitários de saúde (ACS) no acompanhamento, em domicílio, de pacientes em situação de risco (por exemplo, desnutridos, diabéticos, hipertensos, gestantes).

Observa-se que foi implementado na SMSA/BH um sistema de gestão de saúde no plano ambulatorial, organizado a partir das atividades assistenciais ofertadas aos pacientes, vinculadas às áreas de compras, de almoxarifado e de RH.

A informação que alimenta os sistemas de produção, inclusive os de base nacional, advém do processo assistencial. Isso confere qualidade à informação produzida para acompanhamento e planejamento de atividades, além de evitar duplicidade. O sistema tem como subproduto o fornecimento, de forma automática, de todas as informações requeridas pelos sistemas nacionais.

\section{DISCUSSÃO}

Na experiência de Belo Horizonte, a estruturação adequada do acolhimento permitiu uma melhor avaliação da porta de entrada do sistema, o que agilizou o atendimento e possibilitou segurança para os profissionais pela possibilidade de detalhar o atendimento realizado, disponibilizando a informação para outros profissionais (22). A implantação dos módulos Agendamento e Recepção propiciou uma melhor organização do acesso da população às unidades, diminuindo filas de forma drástica. Em algumas unidades, a implantação desses módulos significou a supressão de filas (23). A implantação do módulo Agendamento permitiu que, na programação de atividades da equipe de saúde da família, houvesse espaços para consultas individuais, atividades de grupo (grupos de hipertensos, diabéticos, pré-natal, reuniões de equipe, entre outros) e de promoção de saúde (por exemplo, grupos de caminhadas para idosos, grupos de serestas para pacientes acamados).

O módulo Coleta de material biológico propiciou a redução do tempo de execução de coleta de exames de 2 horas para 40 minutos, bem como uma significativa redução do tempo de acesso ao resultado de exames, de uma média de 15 dias para menos de 2 dias (24). Os módulos Acolhimento e Registro médico permitiram uma melhora da qualidade do atendimento prestado (25), além de propiciar um conjunto de indicadores fundamentais para a organização do processo de trabalho das equipes do PSF (26). O módulo Farmácia, vinculado ao sistema de materiais e ao almoxarifado central, permitiu um melhor controle da dispensação de medicamentos, agilizando processos e melhorando o controle de estoque de medicamentos da unidade (27).

Um estudo realizado pela Escola Nacional de Saúde Pública, no qual foram avaliadas as experiências de implantação do PSF em quatro capitais brasileiras, retratou positivamente a utilização de informação na SMSA/BH, mostrando que os trabalhadores têm acesso a informações e efetivamente fazem uso delas (28). A experiência de Belo Horizonte permitiu uma contribuição significativa da área de informação para a estruturação de informação de qualidade, embasando o trabalho das equipes do PSF e agilizando processos. A implantação do conceito de GRI, estruturador das políticas de informação, contribuiu para esse resultado: está em sua concepção a ideia de que as tecnologias de informação e a própria informação constituem um recurso capaz de qualificar drasticamente processos instituídos (29).

No Brasil, ao longo do tempo, diversas dimensões das organizações passaram por reengenharias de processos, mas em geral são poucos os casos de mudança em relação ao processo de tomada de decisão (30). A GRI oferece instrumentos para revelar como os processos locais podem ser profundamente melhorados com a incorporação de tecnologias de informação. Em nossa experiência, além do uso da informação, os processos as- sistenciais foram remodelados de acordo com diretrizes estratégicas de níveis diretivos, respeitando as especificidades do nível local, o que contribuiu para a consolidação do novo modelo assistencial centralizado no PSF.

Caminhar na direção do uso da informação no SUS-BH tem sido construir, quotidianamente, um novo ambiente que favoreça o uso da informação pelos profissionais de saúde. Foi necessário superar as exigências da "parafernália tecnológica" e das habilidades técnicas. Deve-se ressaltar que esse salto no processo de produção da informação, tanto para a tomada de decisão clínica quanto para a gerencial e estratégica, desagregada por equipe de saúde da família em uma cidade do porte de Belo Horizonte, exigiu um processo avançado de integração entre as áreas de informática e de informação, como já faz parte da história de estruturação do Sistema de Informação em Saúde de Belo Horizonte.

Exigiu também um enorme processo de aquisição de competências no âmbito de gerenciamento de tecnologias de informação, devido à amplitude do parque tecnológico estruturado, ao crescimento exponencial da base de dados e ao rápido processo de incorporação de equipamentos na área de saúde, com todos os consultórios informatizados e uma média de 15 equipamentos por unidade básica, além de um processo de capacitação de profissionais no manejo dessas novas tecnologias, para o manuseio da informação em seu próprio consultório e sua utilização efetiva. É a própria área de informação que se atualiza quanto ao seu potencial, redefinindo suas competências (31).

Belo Horizonte, nessa sua experiência, traz elementos importantes de reflexão sobre as atribuições da área de informática e informação, objeto de discussão de diversos artigos na área (32). No entanto, a utilização da metodologia de estudo de caso, por analisar experiência realizada em uma única realidade, pode limitar as conclusões relativas à aplicação dessa experiência de GRI em outras realidades organizacionais.

Em Belo Horizonte, o conceito de GRI permitiu abordar a complexidade dos aspectos envolvidos na estruturação da área. No entanto, o conceito traz desafios não totalmente experimentados, que remetem aos aspectos da dimensão estratégica da informação: como estruturá-la 
para além das necessidades internas organizacionais? Como impulsionar seu uso em uma dimensão mais geral (4), captando tendências de necessidades sociais, de adequação dos sistemas de saúde aos mutáveis perfis epidemiológicos típicos da transição demográfica? Essa dimensão da GRI não foi tratada na experiência analisada em Belo Horizonte e constitui o principal limite dessa experiência.

A experiência de Belo Horizonte demonstrou os seguintes elementos presentes no conceito de GRI: 1) necessidade de gerenciamento integrado de recursos informacionais, com estruturação de um único locus para gerenciamento das áreas de informação e tecnologias de informação; 2) utilização do potencial advindo do componente tecnologia para reestruturação de processos de trabalho, redimensionando-os a partir da introdução de tecnologias e utilização de informações de forma integrada; e 3) construção de um campo de decisões estratégicas das questões relativas à informação, com esses recursos sendo usados para alcançar objetivos institucionais estratégicos.

O quarto elemento - o único não construído pela experiência analisada refere-se aos aspectos de sistematização e análise de informações externas à organização. Apesar dessa limitação, a discussão advinda da experiência analisada pode contribuir para a estruturação da área de informação em outras realidades organizacionais.

\section{Conclusão}

As tecnologias de informação e a informação, assim como sua incorporação intensa na área de saúde, são atualmente dimensões constitutivas do sistema de informação em Belo Horizonte, com impacto efetivo na área assistencial. Esse processo ocorreu a partir da implantação de uma estrutura moderna de gerenciamento da informação e de sua inserção em um ambiente estratégico, de formulação de grandes políticas do sistema de saúde.

Essa concepção de estruturação da área de informação a partir dos elementos advindos da GRI para a estruturação do SUS em Belo Horizonte significou avanços importantes. A porta de entrada do sistema a partir das equipes do PSF foi requalificada: ocorreu agilização e organização de processos assistenciais, articulando prevenção e ações curativas; a produtividade dos profissionais envolvidos nas atividades foi evidenciada e, muitas vezes, elevada; a articulação entre sistemas administrativos, como almoxarifado e compras, e assistenciais permitiu identificar duplicidades de consumo de itens, exames realizados e datas de vali- dade vencidas e, principalmente, dotou as equipes de PSF de um instrumento de planejamento e monitoramento de suas atividades, tornando-as capazes de se constituírem como coordenadoras da atenção prestada aos pacientes.

Belo Horizonte experimentou as dimensões de gerenciamento da informação em locus único e estratégico, compartilhamento e integração das áreas de tecnologia e de informação e a potência da utilização da informação enquanto recurso organizacional capaz de requalificar a assistência, melhorando a produtividade e agilizando processos.

Atualmente, o sistema de saúde de Belo Horizonte é considerado uma das experiências mais avançadas de estruturação do SUS no país. A área de informação contribuiu de forma significativa para esse processo, identificando com níveis estratégicos de decisão os saltos que poderiam ser alcançados na estruturação do modelo assistencial, de forma dialógica com as equipes do PSF (a partir de um processo de utilização intensiva de tecnologias de informação e disponibilização de informações para a tomada de decisões clínicas e gerenciais). A área de informação, ao experimentar novos conceitos e descortinar novas possibilidades, deu a sua contribuição para a construção de uma experiência de fronteira, como é o SUS de Belo Horizonte.

\section{REFERÊNCIAS}

1. Levitan KB. Information resource(s) management (IRM). ARIST. 1982;17:227-66.

2. Cronin B. Esquemas conceituais e estratégicos para a gerência da informação. Rev Esc Bibliotecon UFMG. 1990;19(2):195-220.

3. Miranda RCR. O uso da informação na formulação de ações estratégicas pelas empresas. Ci Inf. 1999;28(3):286-92.

4. Becker LG. Information resources management (IRM): a revolution in progress. Bull Am Soc Inf Sci. 1980;6(6):26-7.

5. Vieira AS. Gerência de recursos informacionais: $\mathrm{o}$ advento do futuro. Rev Esc Bibliotecon UFMG. 1990;19(2):159-70.

6. Savic D. Evolution of information resources management. JOLIS. 1992;2(3):127-38.

7. Davenport TH. Ecologia da informação. São Paulo: Futura; 1998.

8. Stewart TA. Capital intelectual: a nova vantagem competitiva das empresas. Rio de Janeiro: Campus; 1998.

9. Choo CW. The knowing organization. Oxford: Oxford University Press; 1998.

10. Nonaka I, Takeuchi H. Criação de conhecimento na empresa: como as empresas japone- sas geram a dinâmica da inovação. Rio de Janeiro: Campus; 1998.

11. Rojas MY. Organización de la información: un factor determinante en la gestión empresarial. ACIMED. 2004;12(2):82-4.

12. Melouk S, Raja U, Keskin B. Managing resource allocation and task prioritization decisions in large scale virtual collaborative development projects. IRMJ. 2010;23(2): 53-76.

13. Santos AF, Souza C, Alves HJ, Melo MCB, Santos SF. Structuring of the Brazilian Public Health System and the development of telehealth activities in Brazil. Latin Am J Telehealth. 2009;1(1):5-38.

14. Belo Horizonte. Decreto 12 916/2007. Estabelece a criação da gerência de recursos informacionais. Diário Oficial [do município de Belo Horizonte]. Belo Horizonte, 31 de outubro de 2007, seção 1.

15. Yin R. Estudo de caso: planejamento e métodos. $2^{\mathrm{a}}$ ed. Porto Alegre: Bookman; 2001.

16. Stake RE. Case studies. Em: Denzin NK, Lincoln YS, eds. Handbook of qualitative research. London: Sage; 2000.
17. Belo Horizonte, Secretaria Municipal de Saúde. Portaria interna 201. Cria o comitê gestor da informação da SMSA/BH. Belo Horizonte: Secretaria Municipal de Saúde; 2003.

18. Belo Horizonte, Secretaria Municipal de Saúde. Relatório de gestão 2003. Belo Horizonte: Secretaria Municipal de Saúde; 2003.

19. Belo Horizonte, Secretaria Municipal de Saúde. Relatório de gestão 2005. Belo Horizonte: Secretaria Municipal de Saúde; 2005.

20. Belo Horizonte, Secretaria Municipal de Saúde. Relatório de gestão 2006. Belo Horizonte: Secretaria Municipal de Saúde; 2006.

21. Belo Horizonte, Secretaria Municipal de Saúde. Relatório de gestão 2008. Belo Horizonte: Secretaria Municipal de Saúde; 2008. Disponível em: http:/ / portalpbh.pbh.gov.br/ $\mathrm{pbh} / \mathrm{ecp} / \mathrm{com}$ unidade.do?app=saude. Acessado em 3 de maio de 2010.

22. Hadad SC, Ribeiro CA, Santos AF. Informatização das unidades básicas de saúde de Belo Horizonte: mudanças e aperfeiçoamento dos processos de trabalho e de gestão. Em: Anais do IX Congresso Brasileiro de Informática em 
Saúde; 2004; Ribeirão Preto, Brasil. São Paulo: Sociedade Brasileira de Informática em Saúde; 2004. Pp. 310-4.

23. Santos AF, Campos RT, Santos SF, Dornas Junior G, Costa RB, Ribeiro CA, et al. Avaliação da implantação do prontuário eletrônico na Secretaria Municipal de Saúde de Belo Horizonte. Em: Anais do X Congresso Brasileiro de Informática em Saúde; 2006; Florianópolis, Brasil. São Paulo: Sociedade Brasileira de Informática em Saúde; 2006. Pp. 1525-9.

24. Hadad SC, Cardoso LFA, Campos EC. O processo de informatização dos laboratórios de patologia clínica da rede de saúde de Belo Horizonte. Em: Anais do VII Congresso Brasileiro de Saúde Coletiva; 2003; Brasília, Brasil. Rio de Janeiro: Abrasco; 2003. P. 154, livro I.

25. Sancho LG, Rievrs N, Reis GA, Cirino MGW, Sena E. Avaliação do projeto "Gestão Saúde em Rede": um primeiro olhar do período pósinformatização na perspectiva da gestão. Em: Anais do X Congresso Brasileiro de Informática em Saúde; 2006; Florianópolis, Brasil.
São Paulo: Sociedade Brasileira de Informática em Saúde; 2006. Pp. 186-9.

26. Hadad SC, Braga LC, Cirino MGW, Reis GA. Sistema Gestão Saúde em Rede e o uso de indicadores para monitoramento de saúde. Em: Anais do XI Congresso Brasileiro de Informática em Saúde; 2008; Campos do Jordão, Brasil. São Paulo: Sociedade Brasileira de Informática em Saúde; 2008. Pp. 245-9.

27. Campos RT, Santos SF, Sancho LG, Santos AF, Santos MCF. Incorporação de tecnologias de informação na assistência farmacêutica do Distrito Sanitário Oeste do município de Belo Horizonte (Secretaria Municipal de Saúde de Belo Horizonte). Em: Anais do $8^{\circ}$ Congresso Brasileiro de Saúde Coletiva; 2006; Rio de Janeiro, Brasil. Rio de Janeiro: Abrasco; 2006. P. 186.

28. Almeida PF, Giovanella L, Mendonça MHM, Escorel S. Desafios à coordenação dos cuidados em saúde: estratégias de integração entre níveis assistenciais em grandes centros urbanos. Cad Saude Publica. 2010;26(2):286-98.
29. Gabrieli LC, Cortimiglia MN, Ribeiro JLD. Modelagem e avaliação de um sistema modular para gerenciamento de informação na web. Ci Inf. 2007;36(1):35-53.

30. Khosrow-Pour M. Global, social and organizational implications of emerging information resources management: concepts and applications. Hershey: Information Resources Management Association; 2010.

31. Vitorino EV, Piantola D. Competência informacional - bases históricas e conceituais: construindo significados. Ci Inf. 2009;38(3): $130-41$.

32. Moraes HIS, Gómez MNG. Informação e informática em saúde: caleidoscópio contemporâneo da saúde. Cienc Saude Coletiva. 2007;12(3):553-65.

Manuscrito recebido em 28 de maio de 2010. Aceito em versão revisada em 6 de dezembro de 2010.

ABSTRACT Objective. To report the experience of the City Department of Health in Belo Horizonte (SMSA/BH), state of Minas Gerais, Brazil, with the use of information resources management (IRM) to develop a health information system.

\section{Using information resources management to set up the area of health information: a case analysis}

Methods. In this case study we reviewed documents describing initiatives in the area of information, the mechanisms used to enable these initiatives, and the results achieved with a management system focused on the implementation of an electronic medical records system in the period from 2003 to 2008. This process is described and analyzed from the perspective of IRM.

Results. The system contributed to a change in the health care model, resulting from the electronic integration of 103 basic health units, 9 specialized units, and 503 family health teams, with emphasis on information that was relevant for the family health program.

Conclusions. The following IRM components were effectively implemented as part of the electronic system: creation of a single locus for the areas of information technology and information, potential leveraging of information technology, and establishment of a strategic forum for information-related decision-making. One limitation refers to the use of strategic information for long-term decision-making.

Key words Information systems; medical records systems, computerized; primary health care; Brazil. 